\title{
THORACIC SURGERY IN EARLY LIFE
}

\author{
By R. B. ZACHARY, F.R.C.S. \\ Consultant Paediatric Surgeon, The Children's Hospital, Sheffield
}

The problems of cardio-vascular surgery in infancy cannot be satisfactorily included in this short review, which will deal with thoracic diseases other than those of the heart and great vessels. The problems will be considered under five headings :-

I. Oesophageal atresia and stenosis.

2. Diaphragmatic hernia.

3. Mediastinal cysts and neoplasms.

4. Lung cysts and infections.

5. Deformities of the thorax.

\section{r. Atresia of the Oesophagus}

In practice, only two types of atresia of the oesophagus need consideration, those with a fistula between the trachea and lower end of the oesophagus and those without. . More than 90 per cent. of the cases fall into the first group and most of the rest into the second group. Other types are rare.

In all cases, symptoms arise from swallowed saliva spilling over into the trachea, causing spasms of coughing or cyanosis or a persistently cyanotic hue. It must be the aim of a midwife or practitioner to recognize this condition before giving the first feed, which would swamp the respiratory passages with milk.

The earliest and most important single sign is the drooling of an abnormal amount of saliva from the mouth in the first few hours of life. Hydramnios in the mother and other congenital anomalies in the child (and especially imperforate anus) should also lead to an investigation for atresia of the oesophagus.

A soft rubber catheter is passed through the mouth; a hold up about three or four inches from the mouth is strong evidence in favour of atresia and the infant should be sent to hospital at once. In hospital, proof of obstruction is obtained by an X-ray of an opaque catheter passing through the mouth into the oesophageal pouch. The presence of air in the stomach or bowel proves the presence of a tracheo-oesophageal fistula.

Pre-operative treatment. Gentle suction is applied to a fine soft rubber catheter, passed through the nose and into the oesophageal pouck. If the infant is tilted with the head up, saliva will collect in the pouch and be aspirated. The infont is kept in oxygen, blood is cross-matched anse polythene cannula inserted into the saphenoils vein just before operation, or earlier if the baby is dehydrated. At most, a little 5 per cent. glucose will be needed intravenously; no shist should be given.

Operation. Endotracheal anaesthesia gikes better control than a small, close-fitting mask, büt the latter method is advocated by some. The tube should be short and care must be taken that the end does not lie in a bronchus. The chestegs opened through the right fourth intercostal speee, the lung retracted forwards, a flap of pleura rissed and the azygos vein tied. The upper and Touer ends of the oesophagus are then exposed a ged mobilized, the fistula is divided close to the tracha and this end is oversewn. If at all possible, the fistula should not be used for anastomosis, for is wall is thin and its blood supply poor.'Adequâe mobilization as a rule allows direct suture with single row of interrupted silk sutures. Tension $\mathrm{gn}$ the suture line can be relieved by tacking the upper and lower portions of the oesophagus to adjacept paravertebral fascia, and the anastomosis covered by the pleural flap. A small catheter 3 is left close to the pleural incision and connected go underwater drainage.

The two chief post-operative problems are the prevention of respiratory obstruction and the nourishment of the child. Thick mucus or spissated secretions may narrow the airway गु block it completely, and it is vitally important that partial obstruction should be relieved beforena cyanotic bout occurs. Oxygen and a humied atmosphere help; suction and a laryngoscope should be at the cot side and someone readify available to use them. Pharyngeal suction by the nurse may give some relief.

If the anastomosis is wide, milk feeds can be given by mouth after two or three days, but off there is any narrowing or tension at the sutume line a gastrostomy is performed at the end $\AA_{0} \mathrm{f}$ 
operation and drip feeding commences the following day. Although the infant may take feeds well by mouth after seven to ten days, the gastrostomy tube should be left in situ for six to eight weeks as a safeguard, should a stricture develop.

If there is no fistula the lower end of the oesophagus is usually very short and may project only one or two centimetres above the diaphragm. In these circumstances there is a choice of two procedures to be adopted. One is to enlarge the hiatus in the diaphragm, divide the left gastric artery, and bring up a portion of the stomach into the chest sufficient to allow direct anastomosis of the two ends of the oesophagus. The disadvantage of this method is at once obvious for it involves the creation of an hiatus hernia, and oesophagitis followed by stenosis may occur in exactly the same way as is found in the naturally occurring hiatus hernia. These sequelae are more likely to occur if a small portion of the stomach is brought above the diaphragm than if a large portion is used.

The second technique in these cases with a large gap is to perform a gastrostomy and bring the upper end of the oesophagus out into the neck as an oesophageal fistula. At a later date reconstruction of the oesophagus can be attempted, for example, by using the technique suggested by Waterston in which a portion of the colon is used to supplant the oesophagus.

The mortality rate in cases with fistula should be less than 25 per cent. In those without a fistula it is somewhat higher.

Oesophageal stenosis. Stenosis of the oesophagus is usually post-inflammatory, but a few cases of 'true congenital stenosis have been reported. Some which were thought to be congenital are probably the result of oesophagitis in cases of hiatus hernia. Others are frankly post-inflammatory due to swallowing corrosive fluids. Stenosis following repair of an atresia is well known.

Recognition and treatment of stenosis before it becomes extreme is more important than the technique of dilatation used and success is likely. Occasional short intractable stenoses and a few congenital ones require excision.

\section{Congenital Diaphragmatic Hernia}

A large lateral defect in the diaphragm is five times as frequent on the left side as on the right. In some cases a thin membrane of pleura and peritoneum covers the contents of the hernia, but in others the stomach, bowel and spleen lie in contact with the lung but are not adherent to it. On the right side liver and colon are the chief contents of the hernia.

The main symptoms are cyanosis and dyspnoea, sometimes so severe that it may not be possible to keep the child out of oxygen for more than a few minutes at a time. In these circumstances the baby may take little nourishment by mouth, vomiting may occur and the baby's condition become progressively worse.

Radiographs of the chest show displacement of the heart to the opposite side and intestinal gas shadows in the chest. There is no need to give barium by mouth to demonstrate the hernia.

Treatment is by operation as soon as the condition is recognized. The best route for repair in the newborn infant is through the abdomen because this gives the opportunity of detecting and dealing with any anomaly of the alimentary tract. The gut is gently pulled out of the chest first inserting a catheter through the hole to allow air into the thorax. The bowel is brought out of the abdomen and the defect in the diaphragm repaired by freshening the edges and placing interrupted mattress sutures of nonabsorbable material through the thick muscular rim of the defect and not merely in the thin pleuroperitoneal fold. The anaesthetist expands the lung as the final closure is made, and then begins the trying task of closing the abdominal cavity around a mass of gut which it is not accustomed to contain.

Retro-sternal hernia is rare in infancy; a loop o small or large bowel may be pushed up througf the anterior defect in the diaphragm. Repair is simple and successful.

Hiatus hernia. Herniation at the oesophageal hiatus is of two types. Firstly, there is the protrusion of a large portion of fundus of stomach alongside the oesophagus, the cardia remaining close to the diaphragm. A fold of peritoneum extends part way up the herniated stomach which may be rotated, giving rise to partial obstruction or congestion of the mucosa. The chief symptom is vomiting and the vomitus may contain altered blood. At times bleeding, although not rapid, may be so prolonged as to cause anaemia. Transthoracic replacement of the stomach below the diaphragm and repair of the oesophageal hiatus cures the condition.

The second type of hiatus hernia is found in some cases of cardio-oesophageal reflux. Reflux of gastric contents up the oesophagus occurs in a or small proportion of infants and causes effortless $N$ regurgitation when the child is placed in his cot $\underset{\omega}{\mathrm{N}}$ after a feed. Radiological study of these cases by a barium swallow may reveal nothing more than a lax cardiac orifice.

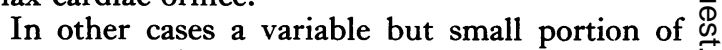
stomach lies above the diaphragm. The vomitus - may contain flecks of altered blood due to oesophagitis and the baby fails to thrive.

It is probably not justifiable to pursue in- 
vestigations further at this stage, unless simple conservative measures fail. These consist of thickening feeds and keeping the baby in a semi-upright position to prevent reflux. In most cases, even in those with obvious stomach above the diaphragm, symptoms will subside and the baby will begin to thrive.

In a small proportion no improvement will occur and oesophagoscopy will show a severe oesophagitis with ulceration just above the cardia and the resulting fibrosis may cause serious stenosis.

Replacement of the stomach below the diaphragm in these cases will sometimes, but not invariably, produce a good result. Failures may be due to extension of gastric mucosa high up the oesophagus.

\section{Mediastinal Cysts and Neoplasm}

The most common mediastinal abnormalities are neurogenic tumours and duplications of the alimentary tract. In both cases symptoms may arise from pressure of the mass on the adjacent trachea, bronchi or lung tissue, giving rise to dyspnoea, cough or signs of a pulmonary infection following partial or complete collapse of a lobe.

Neurogenic tumours consist almost entirely of those arising from the sympathetic nervous system, having their origin in the sympathetic chain in the paravertebral gutter. Neuroblastomas, although arising most commonly in the abdomen in the child, may also start in the chest. They are usually found in the upper part of the posterior mediastinum and a mass in this position should be regarded with grave suspicion. Surgery should always be undertaken in these cases, even though it is impossible to remove the entire tumour, for post-operative irradiation may deal with remaining tissue satisfactorily.

Ganglioneuromas, the well-differentiated type of sympathetic neoplasm, have a much sharper outline on the radiograph than the neuroblastomas. They are benign and slowly growing, and although their removal may be technically difficult, the outlook is good. Some tumours, after removal, are found to contain some portions which are well differentiated and others which are almost completely undifferentiated. They should receive the same radical treatment as the straightforward neuroblastoma.

Duplication of the alimentary tract. These duplications lie in the mediastinum, but may extend outwards into the paravertebral gutter. They are usually lined in whole or in part, by gastric mucosa, the secretions of which fill the duplication, causing it to increase gradually in size and press on adjacent structures. Ulceration of the wall of the cavity is not uncommon and the inflammatory process extends into the adjacent tissues, which may be oesophagus or lung bronchi.

In addition to the symptoms arising from pressure of the mass on the adjacent lung the $\overrightarrow{f e}$ may also be haemoptysis or haematemesis due to this inflammatory process affecting the mucosa gf the bronchus or oesophagus, and the contents the duplication may be discharged through either passage. It is most unusual for the duplication to have a normal communication with the oesophagus. The diagnosis may be made difficult by the inflammatory changes in the adjacent lung tissue, but a barium swallow and a bronchogram will usually reveal the outline of the mass quite well.

The treatment of these tumours is by the removal. This may be exceedingly difficu্t because the duplication may have a close agd possibly extensive attachment to the adjacent oesophagus. If it appears impossible to separ\{te the two muscular coats that of the duplication may be left and after removing most of the cyst the mucous membrane can be peeled off the remaining portion.

\section{Lung Cysts and Infection}

The vast majority of infections of the lung in infancy which come to the attention of the cosidgeons are staphylococcal.

Empyema has become so uncommon as to $\overline{\bar{B}} \mathrm{e}$ unexpected. It is almost always the result of a staphylococcal lung infection and with adequate antibiotic therapy two or three aspirations of t⿳⺈ pus alone may be sufficient to clear the pleuঙral cavities; rib resection and underwater drainage seldom needed.

Staphylococcal infections of the lung are as probably responsible for many cases of cyst-lige cavities in the lung, although these are also fou in other infants who have given no signs ofna respiratory infection. These cavities are often multiple, have a thin, clear outline, and as a rule disappear spontaneously in the course of six to eighteen months.

The chief surgical problem is infants who present, at a few days or weeks of age, with intense respiratory distress, and it is at once clear that there is tension in one side of the thorax. Radinological examination shows extreme translucency in the hemithorax or in its upper part. The differential diagnosis is between tension cyst, tension emphysema and pneumothorax. The latter may be distinguished by the appearance of collapsed lung underneath it and the emphyse may show the lung markings clearly, but the distinction between these three is often difficult. 
If the baby is in extreme distress a wide-bore needle should be inserted between the ribs and connected to an underwater drainage tube. If the relief is not dramatic a tension emphysema is present. Deterioration of the infant's condition, in spite of adequate oxygen therapy, is a strong indication for thoracotomy and removal of the emphysematous lobe as an emergency procedure.

If a spontaneous pneumothorax or a tension cyst is present, the extreme respiratory distress will be relieved at once. In the case of a pneumothorax a further radiograph will show immediate expansion of lung tissue and no treatment other than underwater drainage and antibiotics will be needed.

If a tension cyst shows considerable regression within a few hours and the decreasing flow of air in the drainage bottle indicates that the bronchial check valve mechanism is no longer operative, these cysts may disappear like so many of those without tension.

A large cyst which collapses little on aspiration and continues to produce a strong air flow through the drainage tube requires operation.

\section{Deformities of the Chest Wall}

The chief deformity for which surgery can offer relief is pectus excavatum. Minor degrees of depression of the sternum are not uncommon in infancy, but there are some in whom the depression is considerable and appears to be progressive. In these children, any condition causing dyspnoea is accompanied by a very obvious indrawing of the lower part of the sternum so that recurrent respiratory infections in childhood are likely to accentuate the deformity.

It is unlikely that the deformity will produce cardiac dysfunction, but it is not improbable that the vital capacity of these children will be less than that of others and that there may be some increased risk of respiratory infections as a result of this.

The chief indication for operation is, however, the cosmetic one. It is sometimes stressed that the psychological effect upon the child may be considerable, but these effects can be overcome and should not weigh too heavily in deciding for or against operation.

Since the operation is mainly for cosmetic reasons, the risks must be reasonably small. Provided the operation is done in early childhood, it is a simple procedure and a considerable improvement in appearance is obtained.

The principle of operation is to detach the xiphoid process from the diaphragm and to correct the deformity in the costal cartilages, either by taking wedges out of them or by removing them sub-perichondrially. The position of the sternum is improved by a wedge resection in its upper part and the sternum is held in position by sutures at the site of resection. Sometimes these sutures are sufficient to maintain stability, but at otheep times external fixation by a strong suture passing through the sternum itself is necessary.

Care must be taken to avoid undue prominence of the lower part of the sternum, but if the operation is done at an early age there is a steady improvement in the appearance of the chest even when some deformity remains.

New (Fourth) Edition: 1954

Price 2s. 6d. (2s. 9d., post free)

Published by the

FELLOWSHIP OF POSTGRADUATE MEDICINE

60 Portland Place, London, W.I 